\title{
Congruent and non-congruent hyperball packings related to doubly truncated Coxeter orthoschemes in hyperbolic 3-space
}

\author{
Jenő Szirmai \\ Department of Geometry, Institute of Mathematics, \\ Budapest University of Technology and Economics, \\ Budapest, Hungary \\ email: szirmai@math.bme.hu \\ http://www.math.bme.hu/ szirmai
}

\begin{abstract}
In [17] we considered hyperball packings in 3-dimensional hyperbolic space. We developed a decomposition algorithm that for each saturated hyperball packing has provided a decomposition of $\mathbb{H}^{3}$ into truncated tetrahedra. Thus, in order to get a density upper bound for hyperball packings, it is sufficient to determine the density upper bound of hyperball packings in truncated simplices. Therefore, in this paper we examine the doubly truncated Coxeter orthoscheme tilings and the corresponding congruent and non-congruent hyperball packings. We prove that related to the mentioned Coxeter tilings the density of the densest congruent hyperball packing is $\approx 0.81335$ that is - by our conjecture - the upper bound density of the relating non-congruent hyperball packings, too.
\end{abstract}

\section{Introduction}

In $n$-dimensional hyperbolic space $\mathbb{H}^{n}(\mathrm{n} \geq 2)$ there are 3 types of "balls (spheres)": the classical balls (spheres), the horoballs (horospheres) and the

2010 Mathematics Subject Classification: 52C17, 52C22, 52B15

Key words and phrases: Hyperbolic geometry, hyperball packings, packing density, Coxeter tilings 
hyperballs (hyperspheres).

In this paper we concentrate on the hyperballs and their packings related to the complete Coxeter tilings in 3-dimensional hyperbolic space $\mathbb{H}^{3}$. However, first we survey the previous results related to this topic.

In the hyperbolic plane $\mathbb{H}^{2}$ the universal upper bound of the density of hypercycle packings is $\frac{3}{\pi}$, proved by I. Vermes in [29] and the universal lower bound of the density of hypercycle coverings is $\frac{\sqrt{12}}{\pi}$ determined also by I. Vermes in [30].

In [21] and [22] we analysed the regular prism tilings (simply truncated Coxeter orthoscheme tilings) and the corresponding optimal hyperball packings in $\mathbb{H}^{n}(n=3,4)$, moreover we extended the method - developed in the former paper [22] - to 5-dimensional hyperbolic space (see [23]). In paper [24] we studied the n-dimensional hyperbolic regular prism honeycombs and the corresponding coverings by congruent hyperballs and we determined their least dense (thinnest) covering densities. Furthermore, we formulated conjectures for candidates of the least dense hyperball covering by congruent hyperballs in 3- and 5-dimensional hyperbolic spaces.

In [16] we discussed congruent and non-congruent hyperball packings of the truncated regular tetrahedron tilings. These are derived from the Coxeter simplex tilings $\{p, 3,3\}(7 \leq p \in \mathbb{N})$ and $\{5,3,3,3,3\}$ in 3 - and 5-dimensional hyperbolic spaces, respectively. We determined the densest hyperball packing arrangement and its density with congruent hyperballs in $\mathbb{H}^{5}$ and determined the smallest density upper bounds of non-congruent hyperball packings generated by the above tilings in $\mathbb{H}^{n},(n=3,5)$.

In [15] we dealed with the packings derived by horo- and hyperballs (briefly hyp-hor) packings in $n$-dimensional hyperbolic spaces $\mathbb{H}^{n}(n=2,3)$ which form a new class of the classical packing problems. We constructed in the 2and 3-dimensional hyperbolic spaces hyp-hor packings that are generated by complete Coxeter tilings of degree 1 and we determined their densest packing configurations and their densities. Using also numerical methods we proved that in the hyperbolic plane $(n=2)$ the density of the above hyp-hor packings arbitrarily approximate the universal upper bound of the hypercycle or horocycle packing density $\frac{3}{\pi}$ and in $\mathbb{H}^{3}$ the optimal configuration belongs to the Coxeter tiling $\{7,3,6\}$ with density $\approx 0.83267$. Furthermore, we analyzed the hyp-hor packings in truncated orthoschemes $\{p, 3,6\}(6<p<7, p \in \mathbb{R})$ whose density function attains its maximum for a parameter $p \in[6.05,6.06]$, and the densities are larger that $\approx 0.85397$ if $p$ lies in this interval.

In [14] we proved that if the truncated tetrahedron is regular, then the 
density of the densest packing is $\approx 0.86338$. This is larger than the BöröczkyFlorian density upper bound but our locally optimal hyperball packing configuration cannot be extended to the whole space $\mathbb{H}^{3}$. However, we described a hyperball packing construction, by the regular truncated tetrahedron tiling under the extended Coxeter group $\{3,3,7\}$ with maximal density $\approx 0.82251$.

Recently, (to the best of author's knowledge) the candidates for the densest hyperball (hypersphere) packings in the 3-, 4- and 5-dimensional hyperbolic space $\mathbb{H}^{\mathfrak{n}}$ are derived from regular prism tilings described in [21], [22] and [23].

In [17] we considered hyperball packings in 3-dimensional hyperbolic space. We developed a decomposition algorithm that provides a decomposition of $\mathbb{H}^{3}$ into truncated tetrahedra for each saturated hyperball packing. Therefore, in order to get a density upper bound for hyperball packings, it is sufficient to determine the density upper bound of hyperball packings in truncated simplices.

In [26] we studied hyperball packings related to truncated regular octahedron and cube tilings derived from the Coxeter simplex tilings $\{p, 3,4\}$ $(7 \leq p \in \mathbb{N})$ and $\{p, 4,3\}(5 \leq p \in \mathbb{N})$ in 3-dimensional hyperbolic space $\mathbb{H}^{3}$. We determined the densest hyperball packing arrangement and its density with congruent and non-congruent hyperballs related to the above tilings. Moreover, we proved that the locally densest congruent or non-congruent hyperball configuration belongs to the regular truncated cube with density $\approx 0.86145$. This is larger than the Böröczky-Florian density upper bound for balls and horoballs. We described a non-congruent hyperball packing construction derived from the regular cube tiling under the extended Coxeter group $\{4,3,7\}$ with maximal density $\approx 0.84931$.

In [27] we proved, that the density upper bound of the saturated congruent hyperball (hypersphere) packings related to the corresponding truncated tetrahedron cells is realized in a regular truncated tetrahedra with density $\approx 0.86338$. Furthermore, we proved that the density of locally optimal congruent hyperball arrangement in regular truncated tetrahedron is not monotonically increasing function of the height (radius) of corresponding optimal hyperball, contrary to the ball (sphere) and horoball (horosphere) packings.

In the present paper we study congruent and non-congruent hyperball packings generated by doubly truncated Coxeter orthoscheme tilings in the 3-dimensional hyperbolic space. We prove that the densest congruent hyperball packing belongs to the Coxeter orthoscheme tiling of parameter $\{7,3,7\}$ with density $\approx 0.81335$ (see Theorems 2-3). This density is equal - by our conjecture with the upper bound density of the corresponding non-congruent hyperball arrangements (see Theorem 4 and Conjecture 1). 


\section{Orthoschemes, hyperspheres and their volumes}

An orthoscheme $\mathcal{O}$ in $\mathbb{H}^{n}(\mathrm{n} \geq 2)$ "in classical sense" is a simplex bounded by $n+1$ hyperplanes $H_{0}, \ldots, H_{n}$ such that $H_{i} \perp H_{j}$, for $j \neq i-1, i, i+1$. Or, equivalently, the $n+1$ vertices of $\mathcal{O}$ can be labelled by $A_{0}, A_{1}, \ldots, A_{n}$ in such a way that $\operatorname{span}\left(A_{0}, \ldots, A_{i}\right) \perp \operatorname{span}\left(A_{i}, \ldots, A_{n}\right)$ for $0<i<n-1$.

Geometrically, complete orthoschemes of degree $m=0,1,2$ can be described as follows:

1. For $\mathrm{m}=0$, they coincide with the class of classical orthoschemes introduced by Schläfli. The initial and final vertices, $A_{0}$ and $A_{n}$ of the orthogonal edge-path $A_{i} A_{i+1}, i=0, \ldots, n-1$, are called principal vertices of the orthoscheme.

2. A complete orthoscheme of degree $m=1$ can be constructed from an orthoscheme with one outer principal vertex, one of $A_{0}$ or $A_{n}$, which is simply truncated by its polar plane (see Fig. 1-2).

3. A complete orthoscheme of degree $m=2$ can be constructed from an orthoscheme with two outer principal vertices, $A_{0}$ and $A_{n}$, which is doubly truncated by their polar planes $\operatorname{pol}\left(A_{0}\right)$ and $\operatorname{pol}\left(A_{n}\right)$ (see Fig. 1-2).

For the complete Coxeter orthoschemes $\mathcal{O} \subset \mathbb{H}^{n}$ we adopt the usual conventions and sometimes even use them in the Coxeter case: If two nodes are related by the weight $\cos \frac{\pi}{p}$ then they are joined by a $(p-2)$-fold line for $p=3,4$ and by a single line marked by $p$ for $p \geq 5$. In the hyperbolic case if two bounding hyperplanes of $\mathrm{O}$ are parallel, then the corresponding nodes are joined by a line marked $\infty$. If they are divergent then their nodes are joined by a dotted line.

In the following we concentrate only on dimension 3 and on hyperbolic Coxeter-Schläfli symbol of the complete orthoscheme tiling $\mathcal{P}$ generated by reflections in the planes of a complete orthoscheme $\mathcal{O}$. To every scheme there is a corresponding symmetric $4 \times 4$ matrix $\left(b^{i j}\right)$ where $b^{i i}=1$ and, for $i \neq j \in$ $\{0,1,2,3\}, b^{i j}$ equals to $-\cos \alpha_{i j}$ with all dihedral angles $\alpha_{i j}$ between the faces $\mathrm{H}_{i}, \mathrm{H}_{\mathrm{j}}$ of $\mathcal{O}$.

For example, $\left(\mathrm{b}^{\mathrm{ij}}\right)$ in formula $(1)$ is the so called Coxeter-Schläfli matrix with parameters $(u ; v ; w)$, i.e. $\alpha_{01}=\frac{\pi}{u}, \alpha_{12}=\frac{\pi}{v}, \alpha_{23}=\frac{\pi}{w}$. Now only $3 \leq u, v, w$ come into account (see [7]).

$$
\left(b^{i j}\right)=\left\langle\mathbf{b}^{i}, \mathbf{b}^{j}\right\rangle:=\left(\begin{array}{cccc}
1 & -\cos \frac{\pi}{u} & 0 & 0 \\
-\cos \frac{\pi}{u} & 1 & -\cos \frac{\pi}{v} & 0 \\
0 & -\cos \frac{\pi}{v} & 1 & -\cos \frac{\pi}{w} \\
0 & 0 & -\cos \frac{\pi}{w} & 1
\end{array}\right) .
$$


This 3-dimensional complete (truncated or frustum) orthoscheme $\mathcal{O}=$ $\mathcal{O}(\mathbf{u}, v, w)$ and its reflection group $\mathbf{G}_{\mathfrak{u} v w}$ will be described in Fig. 2, and by the symmetric Coxeter-Schläfli matrix $\left(b^{i j}\right)$ in formula (1), furthermore by its inverse matrix $\left(a_{i j}\right)$ in formula $(2)$.

$$
\begin{aligned}
\left(a_{i j}\right) & =\left(b^{i j}\right)^{-1}=\left\langle\mathbf{a}_{i}, \mathbf{a}_{j}\right\rangle: \\
& =\frac{1}{B}\left(\begin{array}{cccc}
\sin ^{2} \frac{\pi}{w}-\cos ^{2} \frac{\pi}{v} & \cos \frac{\pi}{u} \sin ^{2} \frac{\pi}{w} & \cos \frac{\pi}{u} \cos \frac{\pi}{v} & \cos \frac{\pi}{u} \cos \frac{\pi}{v} \cos \frac{\pi}{w} \\
\cos \frac{\pi}{u} \sin ^{2} \frac{\pi}{w} & \sin ^{2} \frac{\pi}{w} & \cos \frac{\pi}{v} & \cos \frac{\pi}{w} \cos \frac{\pi}{v} \\
\cos \frac{\pi}{u} \cos \frac{\pi}{v} & \cos \frac{\pi}{v} & \sin ^{2} \frac{\pi}{u} & \cos \frac{\pi}{w} \sin ^{2} \frac{\pi}{u} \\
\cos \frac{\pi}{u} \cos \frac{\pi}{v} \cos \frac{\pi}{w} & \cos \frac{\pi}{w} \cos \frac{\pi}{v} & \cos \frac{\pi}{w} \sin ^{2} \frac{\pi}{u} & \sin ^{2} \frac{\pi}{u}-\cos ^{2} \frac{\pi}{v}
\end{array}\right),
\end{aligned}
$$

where

$$
B=\operatorname{det}\left(b^{i j}\right)=\sin ^{2} \frac{\pi}{u} \sin ^{2} \frac{\pi}{w}-\cos ^{2} \frac{\pi}{v}<0 \text {, i.e. } \sin \frac{\pi}{u} \sin \frac{\pi}{w}-\cos \frac{\pi}{v}<0 .
$$

In the following we use the above orthoscheme whose volume is derived by the next Theorem of R. Kellerhals ([8], by the ideas of N. I. Lobachevsky):

Theorem 1 (R. Kellerhals) The volume of a three-dimensional hyperbolic complete orthoscheme $\mathcal{O}=\mathcal{O}(u, v, w) \subset \mathbb{H}^{3}$ can be expressed with the essential angles $\alpha_{01}=\frac{\pi}{u}, \alpha_{12}=\frac{\pi}{v}, \alpha_{23}=\frac{\pi}{w},\left(0 \leq \alpha_{i j} \leq \frac{\pi}{2}\right)$ (Fig. 1.) in the following form:

$$
\begin{aligned}
& \operatorname{Vol}(\mathcal{O})=\frac{1}{4}\left\{\mathcal{L}\left(\alpha_{01}+\theta\right)-\mathcal{L}\left(\alpha_{01}-\theta\right)+\mathcal{L}\left(\frac{\pi}{2}+\alpha_{12}-\theta\right)+\right. \\
& \left.+\mathcal{L}\left(\frac{\pi}{2}-\alpha_{12}-\theta\right)+\mathcal{L}\left(\alpha_{23}+\theta\right)-\mathcal{L}\left(\alpha_{23}-\theta\right)+2 \mathcal{L}\left(\frac{\pi}{2}-\theta\right)\right\}
\end{aligned}
$$

where $\theta \in\left[0, \frac{\pi}{2}\right)$ is defined by:

$$
\tan \theta=\frac{\sqrt{\cos ^{2} \alpha_{12}-\sin ^{2} \alpha_{01} \sin ^{2} \alpha_{23}}}{\cos \alpha_{01} \cos \alpha_{23}},
$$

and where $\mathcal{L}(\mathrm{x}):=-\int_{0}^{\mathrm{x}} \log |2 \sin \mathrm{t}| \mathrm{dt}$ denotes the Lobachevsky function (in J. Milnor's interpretation).

The hypersphere (or equidistant surface) is a quadratic surface at a constant distance from a plane (base plane) in both halfspaces. The infinite body of the hypersphere, containing the base plane, is called hyperball. 
The half hyperball (i.e., the part of the hyperball lying on one side of its base plane) with distance $h$ to a base plane $\beta$ is denoted by $\mathcal{H}_{+}^{\mathrm{h}}$. The volume of the intersection of $\mathcal{H}_{+}^{\mathrm{h}}(\mathcal{A})$ and the right prism with base a 2-polygon $\mathcal{A} \subset \beta$, can be determined by the classical formula of J. Bolyai [2]

$$
\operatorname{Vol}\left(\mathcal{H}_{+}^{\mathrm{h}}(\mathcal{A})\right)=\frac{1}{4} \operatorname{Area}(\mathcal{A})\left[\mathrm{k} \sinh \frac{2 \mathrm{~h}}{\mathrm{k}}+2 \mathrm{~h}\right] .
$$

The constant $k=\sqrt{\frac{-1}{K}}$ is the natural length unit in $\mathbb{H}^{3}$, where $K$ denotes the constant negative sectional curvature. In the following we may assume that $k=1$.

\section{Essential points in a doubly truncated orthoscheme}

Let $A_{0}\left(\mathbf{a}_{0}\right), A_{1}\left(\mathbf{a}_{1}\right), A_{2}\left(\mathbf{a}_{2}\right), A_{3}\left(\mathbf{a}_{3}\right)$ be the vertices of the above complete orthoscheme $\mathcal{O}(\mathfrak{u}, v, w)$ (see Fig. 1,2). In the considered cases the principal vertices $A_{0}$ and $A_{3}$ are outer points $\left(a_{i i}>0\right),(i \in\{0,3\})$.

We distinguish the following main configurations:

1. $A_{3}$ is outer point $\frac{\pi}{u}+\frac{\pi}{v}<\frac{\pi}{2}$, then $\mathbf{a}_{3}\left(\mathbf{a}_{3}\right)=$ JEQ is its polar plane and $A_{0}$ is also outer $\frac{\pi}{v}+\frac{\pi}{w}<\frac{\pi}{2}$, then $\mathrm{a}_{0}\left(\mathbf{a}_{0}\right)=\mathrm{CLH}$ is its polar plane.

1.i $\mathfrak{u}=\mathfrak{w}, \mathrm{F}_{03} \mathrm{~F}_{12}$ is half turn axis, $\mathbf{h}$ is the half turn changing $0 \leftrightarrow 3,1 \leftrightarrow 2$. Here a "half orthoscheme" JQEB ${ }_{13} \mathrm{~F}_{12} \mathrm{~B}_{02} \mathrm{~F}_{03} \mathrm{~A}_{2}$ will be the fundamental domain of $\mathbf{G}_{\mathfrak{u}=w, v}$.

In our calculations we will use the following important lemmas, (see [11] and Fig. 1-2):

Lemma 1 Let $A_{0}$ be an outer principal vertex of the orthoscheme $W_{u v w}$ and let $\mathrm{a}_{0}\left(\mathbf{a}_{0}\right)=\mathrm{CLH}$ be its polar plane where $\mathrm{C}=\mathrm{a}_{0} \cap \mathrm{A}_{0} A_{1}, \mathrm{~L}=\mathrm{a}_{0} \cap \mathrm{A}_{0} A_{2}$, $\mathrm{H}=\mathrm{a}_{0} \cap \mathrm{A}_{0} A_{3}$ whose vectors are the following:

$$
\begin{aligned}
& C(\mathbf{c})=a_{0} \cap A_{0} A_{1} ; \mathbf{c}=\mathbf{a}_{1}-\frac{a_{01}}{a_{00}} \mathbf{a}_{0}, \text { with } \\
& \langle\mathbf{c}, \mathbf{c}\rangle=\frac{\left(a_{11} a_{00}-a_{01}^{2}\right)}{a_{00}}=\left\langle\mathbf{c}, \mathbf{a}_{1}\right\rangle=\frac{\sin ^{2} \frac{\pi}{w}}{\sin ^{2} \frac{\pi}{w}-\cos ^{2} \frac{\pi}{v}}=\frac{a_{11}}{a_{00}} . \\
& L(\mathbf{l})=a_{0} \cap A_{0} A_{2} ; \mathbf{l}=\mathbf{a}_{2}-\frac{a_{02}}{a_{00}} a_{0}, \text { with }
\end{aligned}
$$




$$
\begin{aligned}
\langle\mathbf{l}, \mathbf{l}\rangle=\frac{\left(a_{22} a_{00}-a_{02}^{2}\right)}{a_{00}}=\left\langle\mathbf{l}, \mathbf{a}_{2}\right\rangle=\frac{1}{\sin ^{2} \frac{\pi}{w}-\cos ^{2} \frac{\pi}{v}}=\frac{1}{B a_{00}} . \\
H(\mathbf{h})=a_{0} \cap A_{0} A_{3} ; \mathbf{h}=\mathbf{a}_{3}-\frac{a_{03}}{a_{00}} \mathbf{a}_{0}, \text { with } \\
\langle\mathbf{h}, \mathbf{h}\rangle=\frac{\left(a_{33} a_{00}-a_{03}^{2}\right)}{a_{00}}=\left\langle\mathbf{h}, \mathbf{a}_{3}\right\rangle=\frac{\sin ^{2} \frac{\pi}{v}}{\sin ^{2} \frac{\pi}{w}-\cos ^{2} \frac{\pi}{v}}=\frac{\sin ^{2} \frac{\pi}{v}}{B a_{00}} .
\end{aligned}
$$

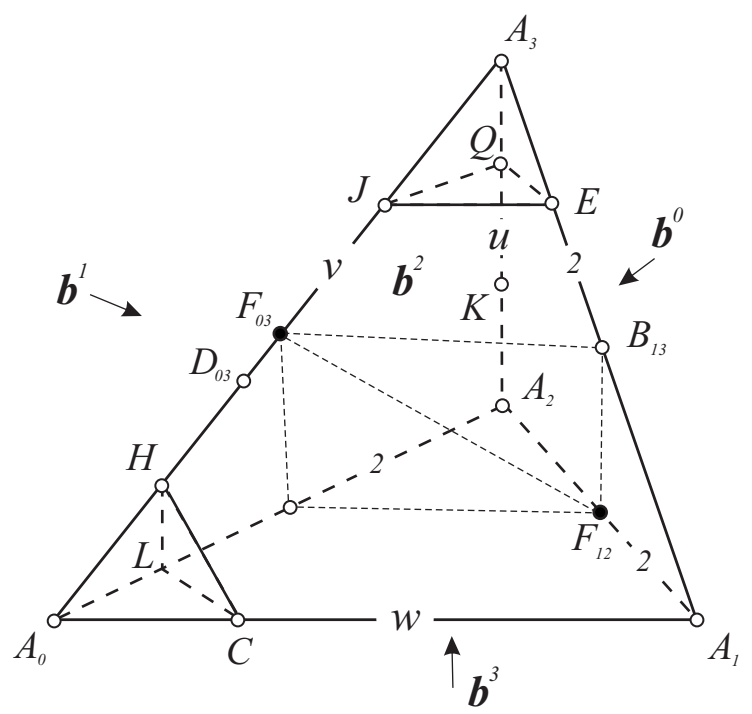

Figure 1: Double truncated complete orthoscheme with essential points

Lemma 2 Let $A_{3}$ be an outer principal vertex of the orthoscheme $\mathcal{O}(u, v, w)$ and let $\mathrm{a}_{3}\left(\mathbf{a}_{3}\right)=\mathrm{JEQ}$ be its polar plane where $\mathrm{J}=\mathrm{a}_{3} \cap \mathrm{A}_{3} A_{0}, \mathrm{E}=\mathrm{a}_{3} \cap \mathrm{A}_{3} A_{1}$, $\mathrm{Q}=\mathrm{a}_{3} \cap \mathrm{A}_{3} \mathrm{~A}_{2}$ whose vectors are the following:

$$
\begin{aligned}
& J(\mathbf{j})=a_{3} \cap A_{3} A_{0} ; \mathbf{j}=\mathbf{a}_{0}-\frac{a_{03}}{a_{33}} \mathbf{a}_{3} \text {, with } \\
& \langle\mathbf{j}, \mathbf{j}\rangle=\frac{\left(a_{00} a_{33}-a_{03}^{2}\right)}{a_{33}}=\left\langle\mathbf{j}, \mathbf{a}_{0}\right\rangle=\frac{\sin ^{2} \frac{\pi}{v}}{\sin ^{2} \frac{\pi}{u}-\cos ^{2} \frac{\pi}{v}}=\frac{\sin ^{2} \frac{\pi}{v}}{B a_{33}} . \\
& E(\mathbf{e})=a_{3} \cap A_{3} A_{1} ; \mathbf{e}=\mathbf{a}_{1}-\frac{a_{13}}{a_{33}} \mathbf{a}_{3} \text {, with }
\end{aligned}
$$




$$
\begin{aligned}
& \langle\mathbf{e}, \mathbf{e}\rangle=\frac{\left(a_{11} a_{33}-a_{13}^{2}\right)}{a_{33}}=\left\langle\mathbf{e}, \mathbf{a}_{1}\right\rangle=\frac{1}{\sin ^{2} \frac{\pi}{u}-\cos ^{2} \frac{\pi}{v}}=\frac{1}{B a_{33}} . \\
& Q(\mathbf{h})=a_{3} \cap A_{3} A_{2} ; \mathbf{q}=\mathbf{a}_{2}-\frac{a_{23}}{a_{33}} \mathbf{a}_{3}, \text { with } \\
& \langle\mathbf{q}, \mathbf{q}\rangle=\frac{\left(a_{22} a_{33}-a_{23}^{2}\right)}{a_{33}}=\left\langle\mathbf{q}, \mathbf{a}_{2}\right\rangle=\frac{\sin ^{2} \frac{\pi}{u}}{\sin ^{2} \frac{\pi}{u}-\cos ^{2} \frac{\pi}{v}}=\frac{a_{22}}{a_{33}} .
\end{aligned}
$$

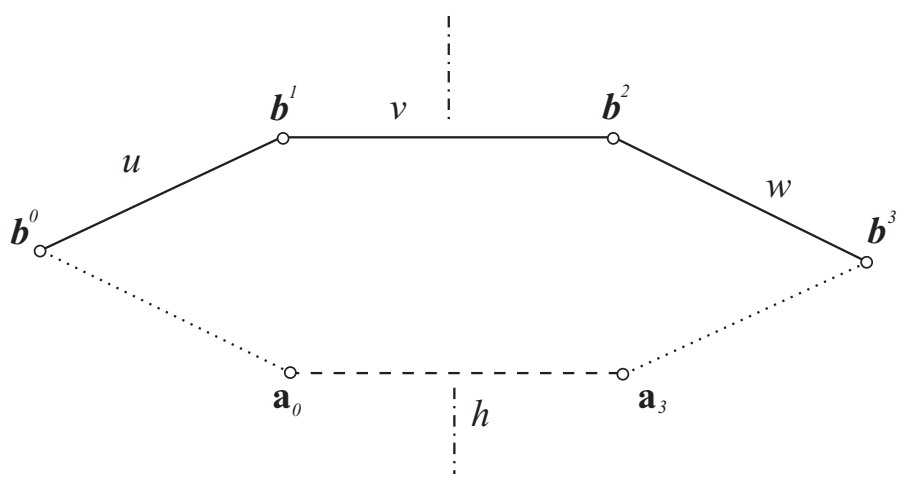

Figure 2:

Especially if $u=w$, the midpoints $F_{03}$ of $J H$ and $F_{12}$ of $A_{1} A_{2}$ can play important roles, since $\mathrm{F}_{03} \mathrm{~F}_{12}$ will be the axis of half turn

$$
\mathbf{h}: 0 \leftrightarrow 3,1 \leftrightarrow 2 \text {, i.e. } A_{0} \leftrightarrow A_{3}, \mathbf{b}^{0} \leftrightarrow \mathbf{b}^{3}, A_{1} \leftrightarrow A_{2}, \mathbf{b}^{1} \leftrightarrow \mathbf{b}^{2} .
$$

(Here $a_{00}=a_{33}$ and $a_{11}=a_{22}$ hold, of course.)

Lemma 3 The midpoints $\mathrm{F}_{03}\left(\mathbf{f}_{03}\right)$ of $\mathrm{JH}$ and $\mathrm{F}_{12}\left(\mathbf{f}_{12}\right)$ of $\mathrm{A}_{1} \mathrm{~A}_{2}$ (see Fig. 1) can be determined by the following vectors:

$$
\begin{aligned}
& \mathbf{f}_{03}=\mathbf{a}_{0}+\mathbf{a}_{3}, \quad\left\langle\mathbf{f}_{03}, \mathbf{f}_{03}\right\rangle=2\left(a_{00}+\mathbf{a}_{03}\right)<0, \\
& \mathbf{f}_{12}=\mathbf{a}_{1}+\mathbf{a}_{2}, \quad\left\langle\mathbf{f}_{12}, \mathbf{f}_{12}\right\rangle=2\left(\mathbf{a}_{11}+\mathbf{a}_{12}\right)<0 .
\end{aligned}
$$

\section{On hyperball packings in a doubly truncated or- thoscheme}

Similarly to the former cases (see [21], [22], [24], [14], [16], [17]) it is interesting to study and to construct locally optimal congruent and non-congruent hyperball packings relating to suitable truncated polyhedron tilings in 3- and 
higher dimensions as well. This study fits into our program to look for the upper bound density of the congruent and non-congruent hyperball packings in $\mathbb{H}^{\mathrm{n}}$.

\subsection{Congruent hyperball packings}

We consider a doubly truncated orthoscheme tiling $\mathcal{T}(\mathcal{O}(u, v, w))$ with Schläfli symbol $\{u, v, w\},\left(\frac{1}{u}+\frac{1}{v}<\frac{1}{2}, \frac{1}{v}+\frac{1}{w}<\frac{1}{2}, 3 \leq u, v, w \in \mathbb{N}\right)$ whose fundamental domain is doubly truncated orthoschem (e.g. CHLA $A_{1} A_{2} E J Q$ in Fig. 1).

Let a truncated orthoscheme $\mathcal{O}(u, v, w) \subset \mathbb{H}^{3}$ be a tile from the above tiling. This truncated orthoscheme can be derived also by truncation from a orthoschem $A_{0} A_{1} A_{2} A_{3}$ with outer essential vertices $A_{0}$ and $A_{3}$. The truncating planes $\mathbf{a}_{0}\left(\mathbf{a}_{0}\right)=\mathrm{CLH}$ and $\mathbf{a}_{3}\left(\mathbf{a}_{3}\right)=$ JEQ are the polar planes of outer vertices $A_{0}$ and $A_{3}$, that can be the ultraparallel base planes of hyperballs $\mathcal{H}_{i}^{s}$ with height $s(i=0,3)$. The distance between the two base planes is $2 h^{03}(u, v, w)=$ $d\left(a_{0}\left(a_{0}\right), a_{3}\left(a_{3}\right)\right)=d(H, J)$ ( $d$ is the hyperbolic distance function).
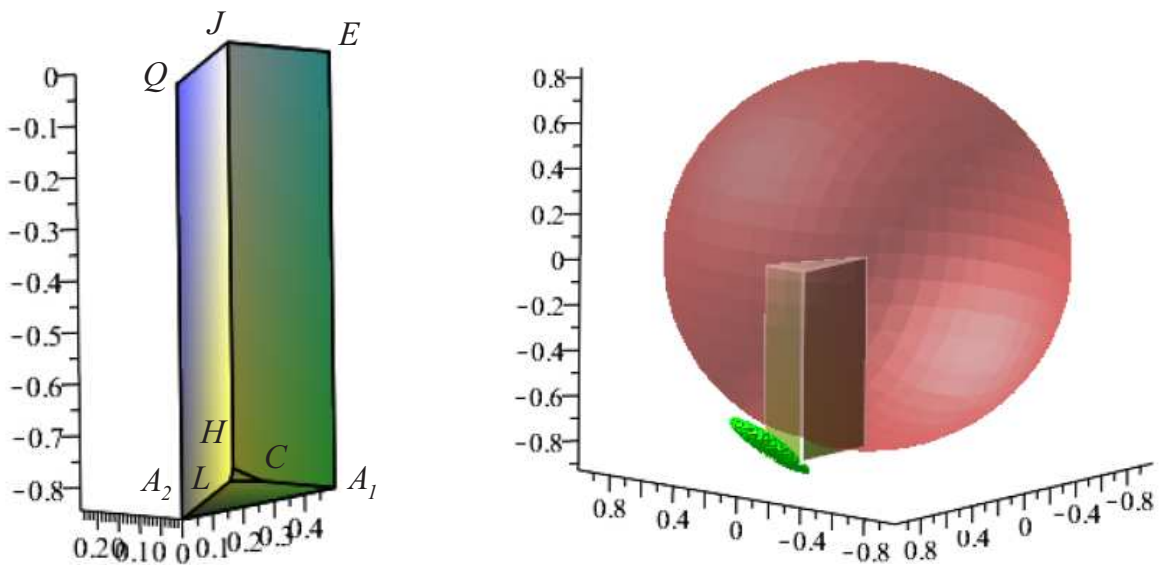

Figure 3: The densest congruent hyperball packing arrangement related to parameters $\{7,3,7\}$ with density $\approx 0.81335$

In this subsection we consider congruent hyperball packings therefore we have to distinguish 2 main cases.

1. Both polar planes are assigned hyperspheres that are congruent with each other therefore the height of a hyperball is at most $h^{03}(u, v, w)$ (see Fig. 1). It is clear, that the heights $h^{0}=h^{3}$ of optimal hyperballs $\mathcal{H}_{i}^{h^{i}}$ 
$(i=0,3)$ is

$$
\begin{aligned}
h & =h^{0}(u, v, w)=h^{3}(u, v, w)= \\
& =\min \left\{h^{03}(u, v, w)=d(H, J) / 2, d\left(Q, A_{2}\right), d\left(C, A_{1}\right)\right\},
\end{aligned}
$$

where $u, v, w$ are suitable given integer parameters. In this case the volume sum of the hyperball pieces lying in the orthoscheme is

$$
\operatorname{Vol}\left(\mathcal{H}^{\mathrm{h}}\left(\mathcal{A}_{0}\right) \cap \mathcal{O}(\mathrm{u}, v, w)\right)+\operatorname{Vol}\left(\mathcal{H}^{\mathrm{h}}\left(\mathcal{A}_{3}\right) \cap \mathcal{O}(\mathrm{u}, v, w)\right)
$$

(see (3)) where $\mathcal{A}_{0}$ is the area of the triangle CLH and $\mathcal{A}_{3}$ is the area of the triangle JEQ.

2. In these cases we consider only one hyperball type:

(a) with base plane $a_{0}\left(\mathbf{a}_{0}\right)=$ CLH. The height of the optimal hyperball $\mathcal{H}_{0}^{\mathrm{h}^{0}}$ is

$$
h^{0}(u, v, w)=\min \left\{2 h^{03}(u, v, w)=d(J, H), d\left(C, A_{1}\right)\right\},
$$

where $u, v, w$ are suitable given integer parameters.

(b) with base plane $a_{3}\left(a_{3}\right)=$ JEQ. The height of the optimal hyperball $\mathcal{H}_{3}^{h^{3}}$ is

$$
h^{3}(u, v, w)=\min \left\{2 h^{03}(u, v, w)=d(J, H), d\left(Q, A_{2}\right)\right\},
$$

where $u, v, w$ are suitable given integer parameters.

Definition 1 The locally density functions $\delta^{i}(\mathcal{O}(u, v, w))$ of the congruent hyperball packings related to $\mathcal{O}(\mathfrak{u}, v, w)$ and the above cases $(i \in\{1,2\})$ are defined by next formulas:

1.

$$
\delta^{1}(\mathcal{O}(u, v, w)):=\frac{\operatorname{Vol}\left(\mathcal{H}^{s}\left(\mathcal{A}_{0}\right) \cap \mathcal{O}(u, v, w)\right)+\operatorname{Vol}\left(\mathcal{H}_{3}^{s}\left(\mathcal{A}_{3}\right) \cap \mathcal{O}(u, v, w)\right)}{\operatorname{Vol}(\mathcal{O}(u, v, w))},
$$

where $0<\mathrm{s} \leq \mathrm{h}, \mathcal{A}_{0}$ is the area of the triangle $\mathrm{CLH}$ and $\mathcal{A}_{3}$ is the area of the triangle JEQ (see (6)).

2.

$$
\delta_{j}^{2}(\mathcal{O}(u, v, w)):=\frac{\operatorname{Vol}\left(\mathcal{H}^{s}\left(\mathcal{A}_{j}\right) \cap \mathcal{O}(u, v, w)\right)}{\operatorname{Vol}(\mathcal{O}(u, v, w))}
$$

where $0<\mathrm{s} \leq \mathrm{h}^{\mathrm{j}}, \mathbf{j} \in\{0,3\}$ (see (7), (8)) and

$$
\delta^{2}(\mathcal{O}(u, v, w)):=\max _{j=0,3}\left\{\delta_{j}^{2}(\mathcal{O}(u, v, w))\right\} .
$$


The distance $s$ of two proper points $X(\mathbf{x})$ and $Y(\mathbf{y})$ is calculated by the formula

$$
\cosh s=\frac{-\langle\mathbf{x}, \mathbf{y}\rangle}{\sqrt{\langle\mathbf{x}, \mathbf{x}\rangle\langle\mathbf{y}, \mathbf{y}\rangle}}
$$

If the parameters $u, v, w$ are given then the lengths of the line segments $A_{1} C$, $\mathrm{A}_{2} \mathrm{Q}$ and $\mathrm{JH}$ can be determined by the machinery of the projective geometry using the Lemmas 1-3 and formula (9):

\section{Lemma 4}

$$
d\left(A_{1}, C\right)=\operatorname{arcosh} \frac{1}{\sqrt{a_{00}}}, d\left(A_{2}, Q\right)=\operatorname{arcosh} \frac{1}{\sqrt{a_{33}}}, d(J, H)=\operatorname{arcosh} \frac{-a_{03}}{\sqrt{a_{00} a_{33}}} .
$$

where $\left(a_{i j}\right)(i, j=0,1,2,3)$ is the inverse of the corresponding Coxeter-Schläfli matrix (see (2)).

In our cases the essential dihedral angles of orthoschemes $\mathcal{O}(u, v, w)$ are the following: $\alpha_{01}=\frac{\pi}{u}, \quad \alpha_{12}=\frac{\pi}{v}, \quad \alpha_{23}=\frac{\pi}{w}$ (see Fig. 1), therefore, the volume $\operatorname{Vol}(\mathcal{O}(u, v, w))$ of the orthoscheme $\mathcal{O}(u, v, w)$ can be determined by Theorem 1. Moreover, the maximal height $h, h^{0}$ or $h^{3}$ of congruent optimal hyperballs and the corresponding volumes of the hyperball pieces can be computed for any suitable fixed integer parameters $u, v, w$ (see (3)). Therefore, the density $\delta^{1}(\mathcal{O}(u, v, w))$ or $\delta_{j}^{2}(\mathcal{O}(u, v, w))(j \in\{1,2\})$ (see Definition 1$)$ depends only on the suitable integer parameters $u, v, w$ of the doubly truncated orthoscheme $\mathcal{O}(u, v, w)$.

\subsubsection{Numerical data of the optimal, congruent hyperball arrange- ments}

First, we illustrate our computation method for given parameters and then we summarize the numerical data of optimal congruent hyperball arrangements for several parameters in Tables 1 and 2 .

Results for parameters $u=7, v=3, w=7$ :

$$
\begin{gathered}
d\left(A_{1}, C\right)=\operatorname{arcosh} \frac{1}{\sqrt{a_{00}}}=d\left(A_{2}, Q\right)=\operatorname{arcosh} \frac{1}{\sqrt{a_{33}}} \approx 1.23469, \\
d(J, H) / 2=\frac{1}{2} \operatorname{arcosh} \frac{-a_{03}}{\sqrt{a_{00} a_{33}}} \approx 1.28517
\end{gathered}
$$


Therefore, the optimal heights in all cases are equal: $h=h^{0}=h^{3}=d\left(A_{1}, C\right)=$ $\mathrm{d}\left(\mathrm{A}_{2}, \mathrm{Q}\right) \approx 1.23469$ (see $\left.(6),(7),(8)\right)$.

$$
\begin{gathered}
\operatorname{Vol}(\mathcal{O}(7,3,7)) \approx 0.38325, \operatorname{Vol}\left(\mathcal{H}^{\mathrm{h}}\left(\mathcal{A}_{0}\right)\right)=\operatorname{Vol}\left(\mathcal{H}^{\mathrm{h}}\left(\mathcal{A}_{3}\right)\right)= \\
=\operatorname{Vol}\left(\mathcal{H}^{\mathrm{h}^{0}}\left(\mathcal{A}_{0}\right)\right)=\operatorname{Vol}\left(\mathcal{H}^{\mathrm{h}^{3}}\left(\mathcal{A}_{3}\right)\right) \approx 0.15586
\end{gathered}
$$

1. Two congruent hyperballs:

$$
\delta^{1}(\mathcal{O}(7,3,7))=\frac{2 \cdot \operatorname{Vol}\left(\mathcal{H}^{h}\left(\mathcal{A}_{0}\right) \cap \mathcal{O}(7,3,7)\right)}{\operatorname{Vol}(\mathcal{O}(7,3,7))} \approx 0.81335
$$

2. One hyperball:

$$
\delta_{j}^{2}(\mathcal{O}(7,3,7))=\frac{\operatorname{Vol}\left(\mathcal{H}^{\mathrm{h}}\left(\mathcal{A}_{\mathbf{j}}\right) \cap \mathcal{O}(7,3,7)\right)}{\operatorname{Vol}(\mathcal{O}(7,3,7))} \approx 0.40668,(j \in\{0,3\})
$$

Remark 1 If $\mathrm{u}=w$ and $\mathrm{h} \geq \mathrm{h}^{0}=\mathrm{h}^{3}$ then $\delta^{2}(\mathcal{O}(\mathrm{u}, \boldsymbol{v}, w))=\frac{1}{2} \delta^{1}(\mathcal{O}(\mathrm{u}, \boldsymbol{v}, w))$ ( see the above example with parameters $u=7, v=3, w=7$ ).

In the following Table we summarize the data of the hyperball packings for some parameters $u, v, w \in \mathbb{N}$, where $\mathcal{A}_{\mathfrak{i}}(i \in\{0,3\})$ is the area of the trigonal face (triangle CHL or EJQ) of the truncated tetrahedron related to the vertex $A_{i}$, cf. Fig. 1 (see (6), (7), (8) and Definition 1). We note here, that the role of the parameters $\mathfrak{u}$ and $w$ is symmetrical therefore we can assume, that $\mathfrak{u} \leq \mathfrak{w}$.

The volume $\operatorname{Vol}(\mathcal{O}(u, v, w))$ can be calculated by Theorem 1 The maximal volume sum $\sum_{i=0,3} \operatorname{Vol}\left(\mathcal{H}^{\mathrm{h}}\left(\mathcal{A}_{\mathfrak{i}}\right)\right)$ of the hyperball pieces lying in $\mathcal{O}(\mathfrak{u}, \boldsymbol{v}, \boldsymbol{w})$ can be computed by the formulas (3), (6), (7), (8) and by the above described computation method for each given possible parameters $u, v, w$. Therefore, the maximal density of the congruent hyperball packings related to every doubly truncated orthoscheme tiling with "two hyperball types" - $\delta^{1}(\mathcal{O}(u, v, w))$ (see Definition 1) - can be computed for each possible parameters.

After careful analysis of the function, finally, we obtain the following

Theorem 2 The density function $\delta^{1}(\mathcal{O}(u, v, w)),\left(\frac{1}{u}+\frac{1}{v}<\frac{1}{2}, \frac{1}{v}+\frac{1}{w}<\frac{1}{2}\right.$. $3 \leq \mathfrak{u}, \boldsymbol{v}, \mathfrak{w} \in \mathbb{N})$ attains its maximum at parameters $\{\mathfrak{u}, \boldsymbol{v}, \boldsymbol{w}\}=\{7,3,7\}$ with density $\delta^{1}(\mathcal{O}(7,3,7)) \approx 0.81335$ (see Table 1$)$. 


\begin{tabular}{|c|c|c|c|c|}
\hline \multicolumn{5}{|c|}{ Table 1, two congruent hyperballs } \\
\hline \hline$\{\mathrm{u}, v, w\}$ & $\mathrm{h}$ & $\operatorname{Vol}(\mathcal{O}(\mathrm{u}, v, w))$ & $\sum_{i=0,3} \operatorname{Vol}\left(\mathcal{H}^{\mathrm{h}}\left(\mathcal{A}_{\mathfrak{i}}\right)\right)$ & $\delta^{1}(\mathcal{O}(\mathrm{u}, v, w))$ \\
\hline$\{7,3,7\}$ & 1.23469 & 0.38325 & 0.31172 & 0.81335 \\
\hline$\{7,3,8\}$ & 0.93100 & 0.41326 & 0.25726 & 0.62251 \\
\hline$\{7,3,9\}$ & 0.76734 & 0.43171 & 0.23355 & 0.54099 \\
\hline$\vdots$ & $\vdots$ & $\vdots$ & $\vdots$ & $\vdots$ \\
\hline$\{7,3,50\}$ & 0.11380 & 0.49016 & 0.06121 & 0.12488 \\
\hline$\vdots$ & $\vdots$ & $\vdots$ & $\vdots$ & $\vdots$ \\
\hline$\{8,3,8\}$ & 0.94946 & 0.44383 & 0.33794 & 0.76143 \\
\hline$\{8,3,9\}$ & 0.78366 & 0.46266 & 0.29474 & 0.63704 \\
\hline$\{8,3,10\}$ & 0.67409 & 0.47536 & 0.26747 & 0.56266 \\
\hline$\vdots$ & $\vdots$ & $\vdots$ & $\vdots$ & $\vdots$ \\
\hline$\{8,3,50\}$ & 0.11668 & 0.52248 & 0.06935 & 0.13274 \\
\hline$\vdots$ & $\vdots$ & $\vdots$ & $\vdots$ & $\vdots$ \\
\hline$\{5,4,5\}$ & 0.88055 & 0.46190 & 0.36007 & 0.77955 \\
\hline$\{5,4,6\}$ & 0.73969 & 0.50747 & 0.37287 & 0.73476 \\
\hline$\{5,4,7\}$ & 0.59326 & 0.53230 & 0.32974 & 0.61947 \\
\hline$\vdots$ & $\vdots$ & $\vdots$ & $\vdots$ & $\vdots$ \\
\hline$\{5,4,50\}$ & 0.07206 & 0.59291 & 0.06350 & 0.10710 \\
\hline$\vdots$ & $\vdots$ & $\vdots$ & $\vdots$ & $\vdots$ \\
\hline$\{4,5,4\}$ & 0.80846 & 0.43062 & 0.31702 & 0.73620 \\
\hline$\{4,5,5\}$ & 0.69129 & 0.49789 & 0.38284 & 0.76893 \\
\hline$\{4,5,6\}$ & 0.53064 & 0.52971 & 0.33597 & 0.63426 \\
\hline$\vdots$ & $\vdots$ & $\vdots$ & $\vdots$ & $\vdots$ \\
\hline$\{4,5,50\}$ & 0.05502 & 0.59318 & 0.05710 & \\
\hline & & & & \\
\hline
\end{tabular}

Similarly to the above case the volume of the doubly truncated orthoscheme can be computed by Theorem 1 but here we use only one hyperball type. Now, the volume $-\max _{\mathfrak{i}=0,3}\left\{\operatorname{Vol}\left(\mathcal{H}^{\mathrm{h}}\left(\mathcal{A}_{\mathfrak{i}}\right)\right\}\right.$ - of the optimal hyperball piece lying in $\mathcal{O}(u, v, w)$ can be computed by the formulas (3), (6), (7), (8) and by the described computation method above for each given possible parameters $u, v, w$. Therefore, the maximal density of the congruent hyperball packings related to a doubly truncated orthoscheme tiling with "one hyperball type" $\delta^{2}(\mathcal{O}(u, v, w))$ (see Definition 1$)$ - can be computed for each possible parameters.

After careful analysis of the function, finally, we obtain the following (see Fig. 3)

Theorem 3 The density function $\delta^{2}(\mathcal{O}(u, v, w)),\left(\frac{1}{u}+\frac{1}{v}<\frac{1}{2}, \frac{1}{v}+\frac{1}{w}<\frac{1}{2}\right.$. $3 \leq u, v, w \in \mathbb{N})$ attains its maximum at parameters $\{u, v, w\}=\{4,6,5\}$ with density $\delta^{2}(\mathcal{O}(4,6,5)) \approx 0.63548$ (see Fig. 3 and Table 2). 


\begin{tabular}{|c|c|c|c|c|c|}
\hline \multicolumn{6}{|c|}{ Table 2, one hyperball } \\
\hline$\{u, v, w\}$ & $h^{0}$ & $h^{3}$ & $\operatorname{Vol}(\mathcal{O}(u, v, w))$ & $\max _{i=0,3}\left\{\operatorname{Vol}\left(\mathcal{H}^{\mathrm{h}^{i}}\left(\mathcal{A}_{i}\right)\right\}\right.$ & $\delta^{2}(\mathcal{O}(u, v, w))$ \\
\hline$\{7,3,8\}$ & 0.93100 & 1.25596 & 0.41326 & 0.16371 & 0.39614 \\
\hline$\{7,3,9\}$ & 0.76734 & 1.27042 & 0.43171 & 0.16543 & 0.38320 \\
\hline : & : & : & : & $\vdots$ & : \\
\hline$\{7,3,50\}$ & 0.11380 & 1.32226 & 0.49016 & 0.18040 & 0.36805 \\
\hline$\vdots$ & $\vdots$ & : & $\vdots$ & $\vdots$ & : \\
\hline$\{8,3,9\}$ & 0.78366 & 0.96206 & 0.46266 & 0.17265 & 0.37316 \\
\hline$\{8,3,10\}$ & 0.67409 & 0.97104 & 0.47536 & 0.17531 & 0.36879 \\
\hline$\vdots$ & : & $\vdots$ & $\vdots$ & $\vdots$ & $\vdots$ \\
\hline$\{8,3,50\}$ & 0.11668 & 1.00753 & 0.52248 & 0.18650 & 0.35695 \\
\hline$\vdots$ & $\vdots$ & : & : & $:$ & : \\
\hline$\{5,4,5\}$ & 1.02221 & 1.02221 & 0.46190 & 0.22942 & 0.49668 \\
\hline$\{5,4,6\}$ & 0.73969 & 1.07541 & 0.50747 & 0.25088 & 0.49437 \\
\hline$\{5,4,7\}$ & 0.59326 & 1.10694 & 0.53230 & 0.26448 & 0.49686 \\
\hline$\vdots$ & $\vdots$ & $\vdots$ & : & : & $\vdots$ \\
\hline$\{5,4,50\}$ & 0.07206 & 1.19054 & 0.59291 & 0.30407 & 0.51284 \\
\hline & : & : & : & : & : \\
\hline$\{4,5,4\}$ & 1.06128 & 1.06128 & 0.43062 & 0.24500 & 0.56895 \\
\hline$\{4,5,5\}$ & 0.69129 & 1.16974 & 0.49789 & 0.29371 & 0.58990 \\
\hline$\{4,5,6\}$ & 0.53064 & 1.22646 & 0.52971 & 0.32284 & 0.60946 \\
\hline$\vdots$ & $\vdots$ & : & $\vdots$ & $\vdots$ & : \\
\hline$\{4,5,50\}$ & 0.05502 & 1.19344 & 0.59318 & 0.30555 & 0.51510 \\
\hline : & : & & : & $\cdot$ & : \\
\hline$\{4,6,4\}$ & 0.88137 & 0.88137 & 0.50192 & 0.30049 & 0.59868 \\
\hline$\{4,6,5\}$ & 0.61415 & 0.97970 & 0.55992 & 0.35582 & 0.63548 \\
\hline$\{4,6,6\}$ & 0.48121 & 1.01251 & 0.58850 & 0.32284 & 0.58711 \\
\hline : & : & : & : & $e^{\prime}$ & : \\
\hline$\{4,6,50\}$ & 0.05138 & 0.88231 & 0.64697 & 0.30100 & 0.46522 \\
\hline
\end{tabular}

\subsection{Non-congruent hyperball packings}

In this subsection we consider non-congruent hyperball packings related to the doubly truncated Coxeter orthoschemes which can be derived, similarly to the above section by truncation from an orthoschem $A_{0} A_{1} A_{2} A_{3}$ with outer essential vertices $A_{0}$ and $A_{3}$. The truncating planes are the polar planes of outer vertices $A_{0}$ and $A_{3}$, which can be the ultraparallel base planes of hyperballs $\mathcal{H}_{\mathfrak{i}}^{s}(\mathfrak{i}=0,3)$ with height $s$. The distance between the two base planes is $2 h^{03}(u, v, w)=d(H, J)$. 
Lemma 5 If $\mathrm{u} \leq w$ then $\mathrm{d}\left(\mathrm{C}, \mathrm{A}_{1}\right) \leq \mathrm{d}\left(\mathrm{Q}, \mathrm{A}_{2}\right)$ where $\mathrm{u}, \boldsymbol{v}, \boldsymbol{w}$ are suitable given integer parameters.

Proof. From Lemma 4 we obtain that $d\left(A_{1}, C\right)=\operatorname{arcosh} \frac{1}{\sqrt{a_{00}}}$ and $d\left(A_{2}, Q\right)=$ $\operatorname{arcosh} \frac{1}{\sqrt{a_{33}}}$ where $\left(a_{i j}\right)(i, j=0,1,2,3)$ is the inverse of the corresponding Coxeter-Schläfli matrix (see $(2))$. From the matrix $\left(a_{\mathfrak{i j}}\right)$ follows that

$$
\begin{aligned}
& \frac{1}{\sqrt{a_{00}}}=1 / \sqrt{-\frac{\cos ^{2} \frac{\pi}{w} \cos ^{2} \frac{\pi}{u}-\cos ^{2} \frac{\pi}{w}+\sin ^{2} \frac{\pi}{v}-\cos ^{2} \frac{\pi}{u}}{\cos ^{2} \frac{\pi}{w}-\sin ^{2} \frac{\pi}{v}}}, \\
& \frac{1}{\sqrt{a_{33}}}=1 / \sqrt{-\frac{\cos ^{2} \frac{\pi}{w} \cos ^{2} \frac{\pi}{u}-\cos ^{2} \frac{\pi}{w}+\sin ^{2} \frac{\pi}{v}-\cos ^{2} \frac{\pi}{u}}{\cos ^{2} \frac{\pi}{u}-\sin ^{2} \frac{\pi}{v}}} .
\end{aligned}
$$

Finally, we obtain the statement of the lemma directly from the above formulas.

We may assume that $u \leq w$ because of the symmetrical role of parameters $u$ and $w$. Therefore, the inequality $d\left(C, A_{1}\right) \leq d\left(Q, A_{2}\right)$ inequality holds.

We have to distinguish 2 different main cases all of which we set up from the optimal congruent hyperball packing configuration described in the former subsection.

1. $h=d(H, J) / 2 \leq d\left(C, A_{1}\right)$ (and so $h \leq d\left(Q, A_{2}\right)$ ).

Both polar planes are assigned hyperspheres. It is clear, that in the "starting configuration" (congruent case) the heights of optimal hyperballs $\mathcal{H}^{\mathrm{h}^{\mathrm{i}}}\left(\mathcal{A}_{\mathrm{i}}\right)(\mathfrak{i}=0,3)$ are equal: $\mathrm{h}=\mathrm{h}^{0}=\mathrm{h}^{3}$.

(a) We consider the hyperball (hypersphere) $\mathcal{H}^{\mathrm{h}^{0}}\left(\mathcal{A}_{0}\right)$ and blow up it keeping the hyperballs $\mathcal{H}^{\mathrm{h}^{3}}\left(\mathcal{A}_{3}\right)$ tangent to it upto this hypersphere touch the plane $A_{1} A_{2} A_{3}$ or the plane EJQ (see Fig. 1). During this expansion the height of hyperball $\mathcal{H}^{h^{0}}$ will be $h^{0}=h+x$ where $x \in\left[0, \min \left\{d\left(C, A_{1}\right)-h, h\right\}\right]$, furthermore the height of hyperball $\mathcal{H}^{\mathrm{h}^{3}}\left(\mathcal{A}_{3}\right)$ will be $\mathrm{h}^{3}=\mathrm{h}-\chi$. $(x=0$ means that the hyperballs are congruent.)

(b) We consider the hyperball $\mathcal{H}^{\mathrm{h}^{3}}\left(\mathcal{A}_{3}\right)$ and blow up it keeping the hyperball $\mathcal{H}^{\mathrm{h}^{0}}\left(\mathcal{A}_{0}\right)$ tangent to it upto this hypersphere touch the plane $A_{0} A_{1} A_{2}$ or the plane CLH (see Fig. 1). During this expansion the height of hyperball $\mathcal{H}^{\mathrm{h}^{3}}\left(\mathcal{A}_{3}\right)$ will be $\mathrm{h}^{3}=\mathrm{h}+\mathrm{x}$ where $x \in\left[0, \min \left\{\mathrm{d}\left(\mathrm{Q}, A_{2}\right)-\mathrm{h}, \mathrm{h}\right\}\right]$, furthermore the height of hyperball $\mathcal{H}^{\mathrm{h}^{0}}\left(\mathcal{A}_{0}\right)$ will be $\mathrm{h}^{0}=\mathrm{h}-\mathrm{x}$. 
2. $h=d\left(C, A_{1}\right),\left(d\left(C, A_{1}\right) \leq d\left(Q, A_{2}\right)\right.$ because $\left.u \leq w\right)$.

In this case we distinguish two subcases:

(a) We blow up the hyperball $\mathcal{H}^{\mathrm{h}^{3}}\left(\mathcal{A}_{3}\right)$ until it touch the plane $\mathrm{A}_{0} \mathrm{~A}_{1} \mathrm{~A}_{2}$ or the hyperball $\mathcal{H}^{\mathrm{h}^{0}}\left(\mathcal{A}_{0}\right)$ (see Fig. 1). During this expansion the height of hyperball $\mathcal{H}^{\mathrm{h}^{0}}\left(\mathcal{A}_{0}\right)$ is $\mathrm{h}^{0}=\mathrm{h}$ constant and the height of hyperball $\mathcal{H}^{\mathrm{h}^{3}}\left(\mathcal{A}_{3}\right)$ is $\mathrm{h}^{3}=\mathrm{h}+\mathrm{x}$ where $\mathrm{x} \in\left[0, \min \left\{\mathrm{d}\left(\mathrm{Q}, \mathrm{A}_{2}\right)-\right.\right.$ $\left.d\left(C, A_{1}\right), d(H, J)-2 h\right\}$. (If $x=0$ then the hyperballs are congruent.)

(b) If in the above situation the hyperball $\mathcal{H}^{\mathrm{h}^{3}}\left(\mathcal{A}_{3}\right)$ first touches the hyperball $\mathcal{H}^{\mathrm{h}^{0}}\left(\mathcal{A}_{0}\right)\left(\mathrm{h}^{3}=\mathrm{d}(\mathrm{H}, \mathrm{J})-\mathrm{h} \leq \mathrm{d}\left(\mathrm{Q}, \mathrm{A}_{2}\right)\right)$ then we can continue the blowing of hyperball $\mathcal{H}^{\mathrm{h}^{3}}\left(\mathcal{A}_{3}\right)$ until this hypersphere touch the plane $A_{0} A_{1} A_{2}$ or the plane CLH (see Fig. 1). During this expansion the height of hyperball $\mathcal{H}^{\mathrm{h}^{3}}\left(\mathcal{A}_{3}\right)$ will be $\mathrm{h}^{3}=\mathrm{d}(\mathrm{H}, \mathrm{J})-$ $h+x$ where $x \in\left[0, \min \left\{d\left(Q, A_{2}\right)-d(H, J)+h, h\right\}\right]$, furthermore the height of hyperball $\mathcal{H}^{\mathrm{h}^{0}}\left(\mathcal{A}_{0}\right)$ will be $\mathrm{h}^{0}=\mathrm{h}-\mathrm{x}$.

We extend this arrangements to images of the hyperballs $\mathcal{H}^{h^{i}}\left(\mathcal{A}_{\mathfrak{i}}\right)(\mathfrak{i} \in\{0,3\})$ by the considered Coxeter group and obtain non-congruent hyperball packing $\mathcal{B}(x)$. Its density is defined by the following

Definition 2 The locally density functions $\delta_{x}^{j}(\mathcal{O}(u, v, w))$ of the non-congruent hyperball packings related to $\mathcal{O}(u, v, w)$ in the above cases $(j \in\{1,2\})$ are defined by the next formulas:

$$
\delta_{\chi}^{j}(\mathcal{O}(u, v, w)):=\frac{\operatorname{Vol}\left(\mathcal{H}^{h^{0}}\left(\mathcal{A}_{0}\right) \cap \mathcal{O}(u, v, w)\right)+\operatorname{Vol}\left(\mathcal{H}^{h^{3}}\left(\mathcal{A}_{3}\right) \cap \mathcal{O}(u, v, w)\right)}{\operatorname{Vol}(\mathcal{O}(u, v, w))},
$$

where $0 \leq \mathrm{h}^{0}, \mathrm{~h}^{3}$ are suitable real parameters related to the above main noncongruent cases (depend on $\times$ parameter) and $\mathcal{A}_{0}$ is the area of the triangle $\mathrm{CLH}$ and $\mathcal{A}_{3}$ is the area of the triangle JEQ (see (6)).

The main problem is to find the maximum of density function $\delta_{x}^{j}(\mathcal{O}(u$, $v, w)$ ) for suitable integer parameters $u, v, w$ where $x \in \mathbb{R}$, and $x \in[0, \min \{$ $\left.\left.\mathrm{d}\left(\mathrm{Q}, A_{2}\right)-\mathrm{d}\left(\mathrm{C}, \mathrm{A}_{1}\right), \mathrm{d}(\mathrm{H}, \mathrm{J})-2 \mathrm{~h}\right\}\right]$ or $\mathrm{x} \in\left[0, \min \left\{\mathrm{d}\left(\mathrm{Q}, \mathrm{A}_{2}\right)-\mathrm{d}(\mathrm{H}, \mathrm{J})+\mathrm{h}, \mathrm{h}\right\}\right]$ (see the above two main cases).

\subsubsection{Numerical data of non-congruent hyperball packing arrange- ments}

First, we illustrate our computation method for given parameters and then we summarize the numerical data of optimal non-congruent hyperball arrange- 
ments for several given parameters in Table 3 .

Results for parameters $u=5, v=4, w=5$ :

$$
\begin{aligned}
\mathrm{d}\left(\mathrm{A}_{1}, \mathrm{C}\right) & =\operatorname{arcosh} \frac{1}{\sqrt{\mathrm{a}_{00}}}=\mathrm{d}\left(\mathrm{A}_{2}, \mathrm{Q}\right)=\operatorname{arcosh} \frac{1}{\sqrt{\mathrm{a}_{33}}} \approx 1.02221, \\
\mathrm{~d}(\mathrm{~J}, \mathrm{H}) / 2 & =\frac{1}{2} \operatorname{arcosh} \frac{-\mathrm{a}_{03}}{\sqrt{\mathrm{a}_{00} \mathrm{a}_{33}}} \approx 0.88055
\end{aligned}
$$

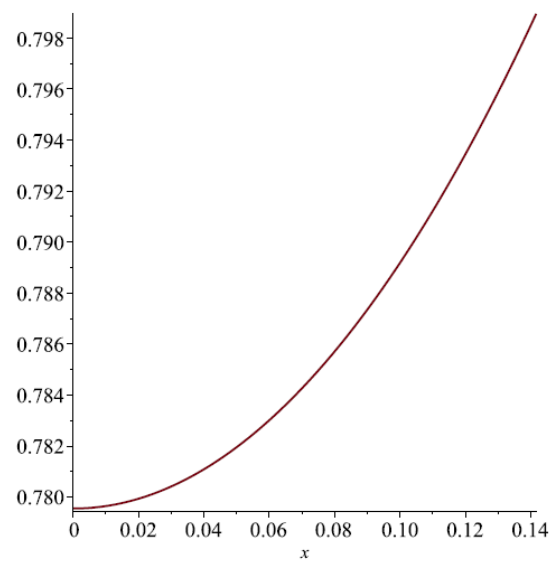

Figure 4: Density function $\delta_{x}^{1}(\mathcal{O}(5,4,5))$ where $x \in\left[0, d\left(C, A_{1}\right)-h \approx 0.14166\right]$

Therefore, the heights in the starting position $(x=0): h=h^{0}=h^{3}=$ $\mathrm{d}(\mathrm{J}, \mathrm{H}) / 2 \approx 0.88055$ (see $(6),(7),(8))$ and $\operatorname{Vol}(\mathcal{O}(5,4,5)) \approx 0.38325$.

We consider the hyperball $\mathcal{H}^{\mathrm{h}^{\mathrm{O}}}\left(\mathcal{A}_{0}\right)$ and blow up it keeping the hyperball $\mathcal{H}^{h^{3}}\left(\mathcal{A}_{3}\right)$ tangent to it until this hypersphere touch the plane $A_{1} A_{2} A_{3}$ (see Fig. 1). During this expansion the height of hyperball $\mathcal{H}^{\mathrm{h}^{0}}$ will be $\mathrm{h}^{0}=\mathrm{h}+\mathrm{x}$ where, furthermore the height of hyperball $\mathcal{H}^{\mathrm{h}^{3}}\left(\mathcal{A}_{3}\right)$ is $\mathrm{h}^{3}=\mathrm{h}-\mathrm{x}$. By the Definition 2 of the density function $\delta_{x}^{1}(\mathcal{O}(5,4,5))$ that it is a strictly increasing function in the intervall $\left[0, d\left(C, A_{1}\right)-h \approx 0.14166\right]$ (see Fig. 4). Thus, the optimal arrangement belongs to the parameter $x^{\text {opt }}=d\left(C, A_{1}\right)-h \approx 0.14166$ :

$$
\begin{gathered}
\delta_{\text {xopt }}^{1}(\mathcal{O}(5,4,5))= \\
=\frac{\operatorname{Vol}\left(\mathcal{H}^{\mathrm{h}^{0}}\left(\mathcal{A}_{0}\right) \cap \mathcal{O}(5,4,5)\right)+\operatorname{Vol}\left(\mathcal{H}^{\mathrm{h}^{3}}\left(\mathcal{A}_{3}\right) \cap \mathcal{O}(5,4,5)\right)}{\operatorname{Vol}(\mathcal{O}(5,4,5))} \approx 0.79895,
\end{gathered}
$$

where $x^{\text {opt }}=\mathrm{d}\left(\mathrm{C}, \mathrm{A}_{1}\right)-\mathrm{h} \approx 0.14166$ and $\mathcal{A}_{0}=\mathcal{A}_{3}=\pi / 2-\pi / 5-\pi / 4$. (see (6)). 

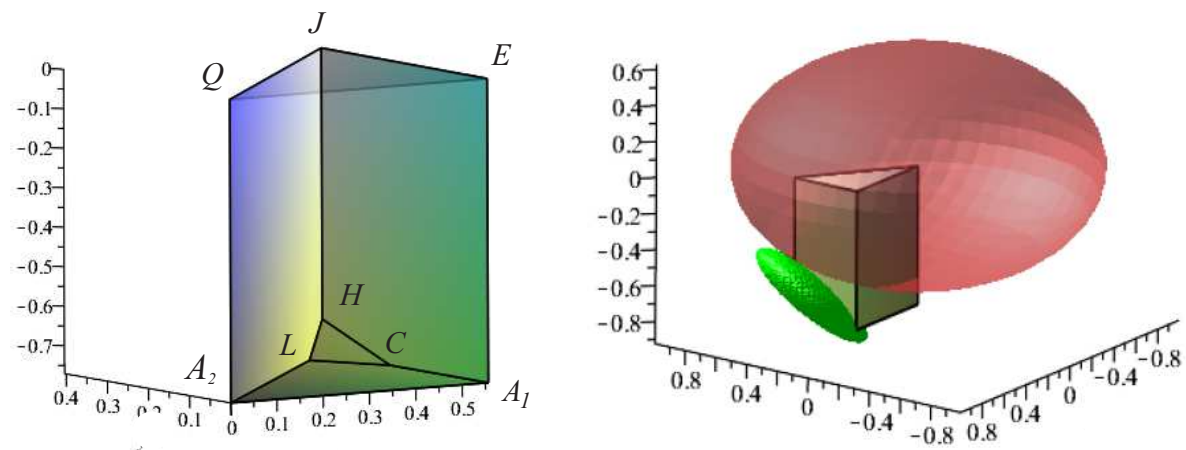

Figure 5: Locally optimal non-congruent hyperball packing configuration related to parameters $\{5,4,5\}$

\begin{tabular}{|c|c|c|c|c|}
\hline \multicolumn{5}{|c|}{ Table 3, allowing non-congruent hyperballs } \\
\hline$\{u, v, w\}$ & $h^{0}$ & $h^{3}$ & $\sum_{i=0,3} \operatorname{Vol}\left(\mathcal{H}^{\mathrm{h}^{i}}\left(\mathcal{A}_{\mathrm{i}}\right)\right)$ & $\delta^{1}(\mathcal{O}(u, v, w))$ \\
\hline$\{7,3,7\}$ & 1.23469 & 1.23469 & 0.31172 & 0.81335 \\
\hline$\{7,3,8\}$ & 0.93100 & 1.25596 & 0.32520 & 0.78690 \\
\hline$\{7,3,9\}$ & 0.76734 & 1.27042 & 0.32892 & 0.76189 \\
\hline$\vdots$ & : & $\vdots$ & : & : \\
\hline$\{7,3,50\}$ & 0.11380 & 1.32226 & 0.23307 & 0.47549 \\
\hline$\vdots$ & $\vdots$ & $\vdots$ & : & : \\
\hline$\{8,3,8\}$ & 0.94946 & 0.94946 & 0.33794 & 0.76143 \\
\hline$\{8,3,9\}$ & 0.78366 & 0.96206 & 0.34107 & 0.73718 \\
\hline$\{8,3,10\}$ & 0.67409 & 0.97104 & 0.33990 & 0.71504 \\
\hline : & : & $\vdots$ & $\vdots$ & : \\
\hline$\{8,3,50\}$ & 0.11668 & 1.00753 & 0.24051 & 0.46032 \\
\hline : & : & : & : & : \\
\hline$\{5,4,5\}$ & 0.73890 & 1.02221 & 0.36903 & 0.79895 \\
\hline$\{5,4,6\}$ & 0.73969 & 0.83611 & 0.39956 & 0.78736 \\
\hline$\{5,4,7\}$ & 0.59326 & 0.90486 & 0.41263 & 0.77517 \\
\hline$\vdots$ & : & : & : & ${ }^{\circ}$ \\
\hline$\{5,4,50\}$ & 0.07206 & 1.19054 & 0.35623 & 0.60082 \\
\hline$\vdots$ & $\vdots$ & $\vdots$ & $\vdots$ & : \\
\hline$\{4,5,4\}$ & 0.55565 & 1.06128 & 0.34184 & 0.79382 \\
\hline$\{4,5,5\}$ & 0.69129 & 0.69129 & 0.38284 & 0.76893 \\
\hline$\{4,5,6\}$ & 0.53064 & 0.77568 & 0.39374 & 0.74331 \\
\hline : & : & : & $\vdots$ & : \\
\hline$\{4,5,50\}$ & 0.05502 & 1.13842 & 0.32720 & 0.55161 \\
\hline$\{5,5,5\}$ & 0.35764 & 0.77537 & 0.41589 & 0.72618 \\
\hline
\end{tabular}


Similarly to the above cases the volume of a doubly truncated orthoscheme can be computed by Theorem 1 and here we allow non-congruent hyperballs. The volume sum $\sum_{i=0,3} \operatorname{Vol}\left(\mathcal{H}^{h^{i}}\left(\mathcal{A}_{\mathfrak{i}}\right)\right)$ of hyperball pieces lying in $\mathcal{O}(u, v, w)$ can be computed by the formulas (3), (6), (7), (8) and by the above described computation method for each given possible parameters $u, v, w$. However, the computations can be contained some subcases, so the determination of the densest hyperball configuration for given parameters $u, v, w$ more complicated than in the congruent cases, as the above example shows.

The maximal density of the congruent hyperball packing related to the doubly truncated orthoscheme tilings with "non-congruent hyperballs" $-\delta_{x}^{i}(\mathcal{O}$ $(u, v, w)$ ) (see Definition (2), $i \in\{1,2\})$ - can be computed for each possible parameters.

Finally, we can formulate only the next Theorem and Conjecture (see Fig. 5):

Theorem 4 The density functions $\delta_{x}^{i}(\mathcal{O}(u, v, w)),\left(\frac{1}{u}+\frac{1}{v}<\frac{1}{2}, \frac{1}{v}+\frac{1}{w}<\frac{1}{2}\right.$. $3 \leq u, v, w \in \mathbb{N}$ and $x \in\left[0, \min \left\{\mathrm{d}\left(\mathrm{Q}, \mathrm{A}_{2}\right)-\mathrm{d}\left(\mathrm{C}, \mathrm{A}_{1}\right), \mathrm{d}(\mathrm{H}, \mathrm{J})-2 \mathrm{~h}\right\}\right]$ or $\mathrm{x} \in$ $\left.\left[0, \min \left\{\mathrm{d}\left(\mathrm{Q}, \mathrm{A}_{2}\right)-\mathrm{d}(\mathrm{H}, \mathrm{J})+\mathrm{h}, \mathrm{h}\right\}\right]\right)$ attain their maximum at $\{\mathrm{u}, \mathrm{v}, \boldsymbol{w}\}=\{7,3,7\}$ with $\mathrm{x}=\mathrm{O}$ (congruent case) among the investigated parameters with density $\delta_{x}^{1}(\mathcal{O}(7,3,7)) \approx 0.81335$ (see Table 3).

Conjecture 1 The density functions $\delta_{x}^{i}(\mathcal{O}(u, v, w)),\left(\frac{1}{u}+\frac{1}{v}<\frac{1}{2}, \frac{1}{v}+\frac{1}{w}<\frac{1}{2}\right.$. $3 \leq u, v, w \in \mathbb{N}$ and $x \in\left[0, \min \left\{\mathrm{d}\left(\mathrm{Q}, \mathrm{A}_{2}\right)-\mathrm{d}\left(\mathrm{C}, \mathrm{A}_{1}\right), \mathrm{d}(\mathrm{H}, \mathrm{J})-2 \mathrm{~h}\right\}\right]$ or $\mathrm{x} \in$ $\left.\left[0, \min \left\{\mathrm{d}\left(\mathrm{Q}, \mathrm{A}_{2}\right)-\mathrm{d}(\mathrm{H}, \mathrm{J})+\mathrm{h}, \mathrm{h}\right\}\right]\right)$ attain their maximum at $\{\mathrm{u}, v, w\}=\{7,3,7\}$ with $x=0$ (congruent case) and with density $\delta^{1}(\mathcal{O}(7,3,7)) \approx 0.81335$ (see Table 3).

\subsubsection{On non-extendable congruent hyperball packings to param- eters $\{p, 3, p\}(6<p<7, p \in \mathbb{R})$}

We can construct infinitely many congruent and non congruent hyperball configuration whose densities are locally larger then the Böröczky-Florian density upper bound $(\approx 0.85328)$. Now, we describe only one, congruent locally dense hyperball arrangement related to parameters $\{p, 3, p\}(6<p<7, p \in \mathbb{R})$.

The computation method described in the former sections is suitable to determine the densities of congruent hyperball packings for real parameters $(6<p<7, p \in \mathbb{R})$ as well. To each such $p$ parameter belongs a doubly truncated orthoscheme and therefore we can determine similarly to the above cases the corresponding maximal density of its optimal congruent hyperball packing. But these packings can not be extended to the 3-dimensional space. 
Analysing these non-extendable packings for these parameters $\mathrm{p}$ we obtain the following (see Fig. 6).

Theorem 5 The function $\delta^{1}(\mathcal{O}(u=p, v=3, w=p)),\left(\frac{1}{u}+\frac{1}{v}<\frac{1}{2}, \frac{1}{v}+\frac{1}{w}<\frac{1}{2}\right.$. $3 \leq u, v, w \in \mathbb{N}$, see Definition 1) is attained its maximum for the parameter $\mathrm{p}_{\mathrm{opt}} \approx 6.05061$ and the density is $\approx 0.85461$. That means that these locally optimal hyperball configurations provide larger density than the Böröczky-Florian density upper bound $(\approx 0.85328)$ for ball and horoball packings $[4]$.

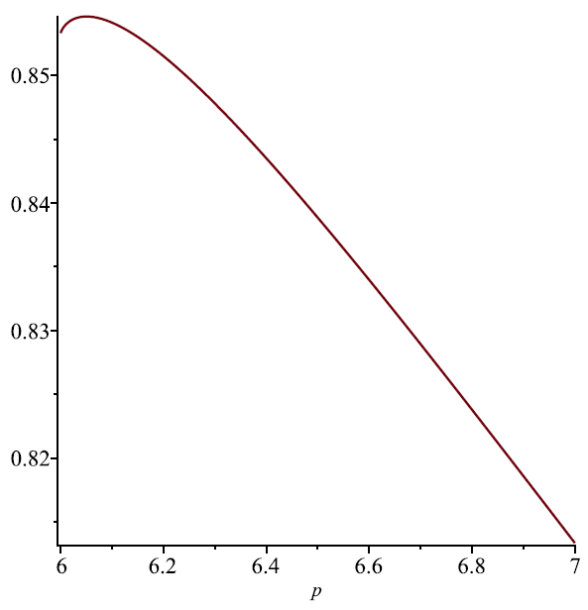

Figure 6: Locally optimal non-extendable congruent hyperball packing configuration related to parameters $\{p, 3, p\}(6<p<7, p \in \mathbb{R})$

In hyperbolic spaces $\mathbb{H}^{n}(n \geq 3)$ the problems of the densest horoball and hyperball packings have not been settled yet, in general (see e.g. [9], [19], [20]). Moreover, the optimal sphere packing problem can be extended to the other homogeneous Thurston geometries, e.g. Nil, Sol, $\widetilde{\mathbf{S L}_{2} \mathbf{R}}$. For these nonEuclidean geometries only very few results are known (e.g. [25] and the references given there).

By the above investigation we can say that the revisited Kepler problem keep several interesting open questions. Detailed studies are the objective of ongoing research. Applications of the above projective method seem to be interesting in (non-Euclidean) crystallography as well. 


\section{References}

[1] K. Bezdek, Sphere Packings Revisited, Eur. J. Combin., 27/6 (2006), 864-883.

[2] J. Bolyai, Appendix, Scientiam spatii absolute veram exhibens, Marosvásárhely, (1831).

[3] K. Böröczky, Packing of spheres in spaces of constant curvature, Acta Math. Acad. Sci. Hungar., 32 (1978), 243-261.

[4] K. Böröczky, A. Florian, Über die dichteste Kugelpackung im hyperbolischen Raum, Acta Math. Acad. Sci. Hungar., 15 (1964), 237-245.

[5] G. Fejes Tóth, G. Kuperberg, W. Kuperberg, Highly Saturated Packings and Reduced Coverings, Monatsh. Math., 125/2 (1998), 127-145.

[6] L. Fejes Tóth, Regular Figures, Macmillan (New York), 1964.

[7] H. -C. Im Hof, Napier cycles and hyperbolic Coxeter groups, Bull. Soc. Math. Belgique, 42 (1990), 523-545.

[8] R. Kellerhals, On the volume of hyperbolic polyhedra, Math. Ann., 285 (1989), 541-569.

[9] R. T. Kozma, J. Szirmai, New Lower Bound for the Optimal Ball Packing Density of Hyperbolic 4-space, Discrete Comput. Geom., 53/1 (2015), 182-198, DOI: 10.1007/s00454-014-9634-1.

[10] E. Molnár, The Projective Interpretation of the eight 3-dimensional homogeneous geometries, Beitr. Algebra Geom., 38/2 (1997), 261-288.

[11] E. Molnár, J. Szirmai, Top dense hyperbolic ball packings and coverings for complete Coxeter orthoscheme groups, Publications de l'Institut Mathématique, 103 (117) (2018), 129-146, DOI: 10.2298/PIM1817129M, arXiv: 1612.04541.

[12] M. Stojanović, Coxeter Groups as Automorphism Groups of Solid Transitive 3-simplex Tilings, Filomat, 28/3 (2014), 557-577, DOI 10.2298/FIL1403557S.

[13] M. Stojanović, Hyperbolic space groups and their supergroups for fundamental simplex tilings, Acta Math. Hungar., 153/2 (2017), 276-288, DOI: $10.1007 / \mathrm{s} 10474-017-0761-\mathrm{z}$. 
[14] J. Szirmai, Hyperball packings in hyperbolic 3-space, Mat. Vesn., 70/3 (2018), 211-221.

[15] J. Szirmai, Packings with horo- and hyperballs generated by simple frustum orthoschemes, Acta Math. Hungar., 152/2 (2017), 365-382, DOI:10.1007/s10474-017-0728-0.

[16] J. Szirmai, Density upper bound of congruent and non-congruent hyperball packings generated by truncated regular simplex tilings, Rendiconti del Circolo Matematico di Palermo Series 2, 67 (2018), 307-322, DOI: 10.1007/s12215-017-0316-8, arXiv:1510.03208.

[17] J. Szirmai, Decomposition method related to saturated hyperball packings, Ars Math. Contemp., 16 (2019), 349-358.

[18] J. Szirmai, The optimal ball and horoball packings to the Coxeter honeycombs in the hyperbolic d-space, Beitr. Algebra Geom., 48/1 (2007), $35-47$.

[19] J. Szirmai, Horoball packings to the totally asymptotic regular simplex in the hyperbolic n-space, Aequat. Math., 85 (2013), 471-482, DOI: 10.1007/s00010-012-0158-6.

[20] J. Szirmai, Horoball packings and their densities by generalized simplicial density function in the hyperbolic space, Acta Math. Hungar., 136/1-2 (2012), 39-55, DOI: 10.1007/s10474-012-0205-8.

[21] J. Szirmai, The p-gonal prism tilings and their optimal hypersphere packings in the hyperbolic 3-space, Acta Math. Hungar., 111 (1-2) (2006), $65-76$.

[22] J. Szirmai, The regular prism tilings and their optimal hyperball packings in the hyperbolic n-space, Publ. Math. Debrecen, 69 (1-2) (2006), 195207.

[23] J. Szirmai, The optimal hyperball packings related to the smallest compact arithmetic 5-orbifolds, Kragujevac J. Math. 40 (2) (2016), 260-270, DOI:10.5937/KgJMath1602260S.

[24] J. Szirmai, The least dense hyperball covering to the regular prism tilings in the hyperbolic n-space, Ann. Mat. Pur. Appl. 195/1 (2016), 235-248, DOI: $10.1007 / \mathrm{s} 10231-014-0460-0$. 
[25] J. Szirmai, A candidate for the densest packing with equal balls in Thurston geometries, Beitr. Algebra Geom., 55/2 (2014), 441- 452, DOI: 10.1007/s13366-013-0158-2.

[26] J. Szirmai, Hyperball packings related to cube and octahedron tilings in hyperbolic space, Contributions to Discrete Mathematics, (to appear), (2020).

[27] J. Szirmai, Upper bound of density for packing of congruent hyperballs in hyperbolic 3-space, Submitted manuscript, (2019).

[28] I. Vermes, Über die Parkettierungsmöglichkeit des dreidimensionalen hyperbolischen Raumes durch kongruente Polyeder, Studia Sci. Math. Hungar., 7 (1972), 267-278.

[29] I. Vermes, Ausfüllungen der hyperbolischen Ebene durch kongruente Hyperzykelbereiche, Period. Math. Hungar., 10/4 (1979), 217-229.

[30] I. Vermes, Über reguläre Überdeckungen der Bolyai-Lobatschewskischen Ebene durch kongruente Hyperzykelbereiche, Period. Math. Hungar., 25/3 (1981), 249-261. 